\title{
Una nueva mirada a la Educación Especial en Cuba a partir del concepto de discapacidad según la CIF
}

\section{Mirtha Leyva Fuentes}

\section{Cuba}




\section{Resumen}

La aprobación en la 54 a. Asamblea Mundial de la Salud en el 2001 de la Clasificación Internacional del Funcionamiento de la Discapacidad y de la Salud (CIF) originó el nuevo concepto de Discapacidad y la nueva Clasificación que se considera hoy como centro para la Familia Internacional de las Clasificaciones junto a la CIE-10. La CIF contiene subyacentes los criterios teóricos de un modelo bio-psico- social que entró en contradicción, entre otros, con el concepto de discapacidad vigente hasta ese momento y asociado a la antigua Clasificación conocida como la CIDDM. Los cambios en el modo de pensar sobre las personas con discapacidad y los servicios y apoyos que se les proveen son la consecuencia más significativa que trae aparejada la CIF. En Cuba éstos están por darse aún, por lo que nosotros hemos centrado nuestro trabajo en los cambios que han de ocurrir dentro de la práctica educativa cubana y más específicamente en la Educación Especial en Cuba. En este sentido proponemos hacer una nueva mirada a las actuaciones, acciones y finalidades que desde la escuela, especial o no, se realizan o han de implementarse y que luego se traducirán en mayor calidad de vida; a partir del análisis de los resultados en la actividad personal y la participación social de los individuos. Para ello, entendemos que ha de verse como meta el alcance del nivel más mínimo posible de limitaciones y restricciones acorde con la deficiencia de que se trate.

Esta investigación es teórica y realiza un análisis de cómo han de interpretarse y traducirse estos cambios en la práctica educativa cubana.

Palabras Clave: Cuba. Educación Especial. Discapacidad. CIF. 


\section{Introducción.}

"El desarrollo de las concepciones sobre las personas tiene su paralelismo en el desarrollo de los términos utilizados para denominarlas, aunque la atención y tratamiento de las personas con discapacidad no es precisamente una cuestión de terminología. Sin embargo, la terminología es el reflejo de los cambios en las concepciones, modos de enfocar, actitudes, y entendimiento del problema"(Verdugo, 1995) Estando de acuerdo con este modo de ver el problema conceptual que nos muestra Verdugo, nos hemos visto en la necesidad de hacer este trabajo donde pretendemos tocar aspectos esenciales que han de transformarse en el campo de los modos de encauzar actitudes para comprender el problema de la discapacidad, no en el orden teórico solamente sino también en el práctico, es decir, en lo que se refiere a cómo ha de llevarse esto a las prácticas educativa y social.

La aparición de la CIF ha traído aparejado, en el ámbito internacional, un cambio en el modo de pensar sobre las personas con discapacidad y los servicios y apoyos que se les proveen. Todo cambio al nivel teórico de la Ciencia, conlleva a su vez a cambios al nivel práctico y viceversa; nosotros hemos centrado nuestro trabajo en los cambios que han de ocurrir dentro de la práctica educativa cubana y más específicamente en la Educación Especial en Cuba.

En este sentido proponemos hacer una nueva mirada a las actitudes, actuaciones, acciones que manifiestan los profesionales implicados, así como a los cambios que desde la escuela especial o no, han de implementarse y que luego se traducirán en mayor calidad de vida; a partir del análisis de los resultados en la actividad personal y la participación social de los individuos. Para ello, entendemos que ha de verse como meta el alcance del nivel más mínimo posible de limitaciones y restricciones acorde con la deficiencia de que se trate.

A pesar del importante papel de las actitudes hacia las personas con discapacidad para lograr una integración social real, todavía éste continúa siendo un campo en el que arar, se necesitan estudios acerca de este lado del fenómeno social de cómo entender y tratar la discapacidad, así como en los planteles escolares se necesitaría insertar (dirigido a profesionales y escolares) actividades, objetivos y contenidos que permitan evaluar y a mejorar las actitudes hacia las personas con discapacidad. 
Hemos estructurado nuestro trabajo en diferentes acápites que nos permiten poder mostrar sin dejar lugar a dudas cómo ha de ser crítica esa mirada a la Educación Especial en Cuba y cuál sería entonces la nueva misión para el logro de los cambios que han de producirse en los servicios y apoyos a proveer para las personas con diferentes niveles de discapacidad. Es por ello que nuestro artículo quedará estructurado de la siguiente manera:

- Estructura del Sistema Nacional Educativo Cubano

- La Educación Especial en Cuba y su situación respecto a la visión internacional.

- Evolución conceptual de la Discapacidad, la CIF y el nuevo concepto.

- La Discapacidad, una nueva visión y una nueva escuela.

\section{Estructura del Sistema Nacional Educativo Cubano}

Antes de presentar la estructura del sistema educativo cubano, creemos necesario señalar algunas particularidades. En Cuba no existen centros de estudios privados a ningún nivel. El acceso a los de enseñanza obligatoria es sólo atendiendo al lugar donde se encuentre el domicilio del escolar, excepto en los casos de asistencia a determinado tipo de escuela especial y nunca a los aspectos de raza, religión o estatus social. No sólo caracteriza el sistema la gratuidad, sino también el diseño curricular de cada una de las educaciones. Es decir: los programas, planes y material de estudio para cada una de ellas es idéntico en cualquier zona o rincón del país. La política educacional es una sola y se dicta y cumple atendiendo al nivel jerárquico en que se han organizado las Instancias de Dirección.

\section{ESTRUCTURA DEL SISTEMA NACIONAL EDUCATIVO CUBANO}

1. EDUCACIÓN GENERAL, POLITÉCNICA Y LABORAL (niveles a alcanzar: primario y medio)

$\diamond \quad$ Educacion Infantil:

- Educación Preescolar

Círculos Infantiles (1-5 años, organizada en 4 años de vida: $2^{\circ} 3^{\circ}, 4^{\circ}$ y

$\left.5^{\circ}\right)$

Grado Preescolar ( $6^{\circ}$ año, en Círculos infantiles o en escuelas primarias, un curso)

- Educación Primaria (nivel: primario)

* escuelas primarias (seis grados)

- Educación Especial (nivel: primario y medio) 


$$
\begin{aligned}
& \text { * de sordos e hipoacústicos } \\
& * \text { de ciegos y débiles visuales } \\
& * \text { de Limitados físicos-motores } \\
& \quad * \text { de retraso mental } \\
& \quad * \text { de problemas de conducta } \\
& \diamond \quad \text { Educación Media: } \\
& \text { - Secundaria Básica (tres grados) } \\
& \text { - Preuniversitario (tres grados) }
\end{aligned}
$$

2. EDUCACIÓN TÉCNICA Y PROFESIONAL (nivel: medio)

Escuelas de Oficios

Institutos Politécnicos

3. EDUCACIÓN DE ADULTOS (nivel: medio)

Escuela de Idiomas

Secundaria Obrera

Facultad Obrera

4. EDUCACIÓN SUPERIOR (nivel: superior)

Institutos Pedagógicos

Universidades e Institutos

Sistema de Postgrado

OTRA VIA DE FORMACIÓN Y PERFECCIONAMIENTO DEL PERSONAL PEDAGÓGICO (nivel: medio)

Escuelas pedagógicas de formación emergente (nivel: medio)

Las instancias o estructuras de dirección únicas y en orden jerárquico ascendente son:

$\diamond$ De centro (uno en cada plantel)

$\diamond \quad$ Municipal (Dirección Municipal de Educación, DME 169)

$\diamond \quad$ Provincial (Dirección Provincial de Educación, DPE 15)

$\diamond \quad$ Nacional (Ministerio de Educación, MINED, 1)

Por supuesto, para nuestros análisis perspectivos y propuesta de cambios vamos a centrarnos en la Educación General politécnica y laboral como marco referencial más general, dentro de ella nos referiremos a la Educación Infantil y más específicamente en lo que concierne a la Educación Especial.

\section{La Educación Especial en Cuba y su situación respecto a la visión actual internacional.}

En la literatura relacionada con la evolución de la Educación Especial se reconocen tres momentos o etapas importantes que sintetizan su devenir histórico conocidas como eras: 
1. La era de las instituciones

2. La era de las escuelas especiales o centros de Educación Especial. Y

3. La era de la integración escolar o normalización. (“mainstream”)

Las dos primeras son denominadas por múltiples autores como "segregacionistas" por aducir que los sujetos eran/son segregados de los centros educativos ordinarios. Asimismo reconocen el surgimiento de la tercera era como resultante de un proceso casi simultáneo de varios países, a consecuencia del principio de normalización ("mainstream"- normalización: crear entornos normales) elaborado en 1969 por Nirje (Suecia) y Bank-Mikkelsen (Dinamarca) y en 1971 por Wolfensberger, para desinstitucionalizar y proceder a la integración (ubicar alumnos en contextos menos restrictivos). Con este principio desaparece la clasificación de algunas personas las categorías de educables y entrenables a una sola, la de "educables".

A pesar de esto, Cuba que aún tiene una Educación Especial predominantemente del tipo correspondiente a la segunda era, se ha empeñado en demostrar que no cabemos en la categoría de "segregacionistas". En distintos documentos y artículos de varios autores cubanos, entre los que se destaca Rafael Bell/ Director nacional de Educación Especial del MINED, se han utilizado disímiles argumentos científicos y bien fundados que argumentan esta aseveración (Bell, 1996, 1997, 2001; Gómez, 1998, 1999, 2001)

1. La igualdad asegurada desde el derecho mismo a la educación que tienen todas las personas sin distinción, así reflejada en la Constitución de la República de Cuba y en el Código de la Niñez y la Juventud.

2. La activa y creciente participación de las Asociaciones cubanas para personas discapacitadas (ANSOC, ACLIFIM, ANCI) de conjunto con todo el pueblo y con el respaldo de organismos e instituciones del estado en importantes programas sociales, científicos y culturales, como muestra de unión sin excluidos ni excluyentes.

3. Se concibe la Educación Especial a partir de un postulado de L.S. Vigotsky: “...en el desarrollo de la personalidad de las personas con deficiencias actúan las mismas leyes que en el resto de los niños." 
4. Al igual que Vigotsky reconocemos como problema práctico de la escuela especial:

- Planteamientos de objetivos comunes con la educación general.

- Peculiaridad de los medios para alcanzar los objetivos planteados.

- Reconocimiento del carácter creador de la escuela especial.

5. El nuevo concepto de Educación Especial en Cuba, aparecido en la década del 90 y entendida como: "Sistema de escuelas, modalidades de atención, recursos, ayudas, servicios de orientación y capacitación, puestos a disposición de los alumnos en grupos de riesgo, con necesidades educativas especiales, sus familias, educadores y el entorno en general" (Bell,1994)

6. Las escuelas especiales están incluidas dentro de la red escolar del Sistema Educativo Cubano, todas son estatales, con alto reconocimiento social y atención priorizada, con acceso y gratuidad para todos.

7. Las escuelas especiales cubanas desarrollan un sistema permanente de actividades infantiles y juveniles para todos como pioneriles, deportivas, culturales y sociales, tanto en el marco estrecho como en el amplio comunitario conjuntamente con los alumnos de la enseñanza general.

8. Las escuelas especiales cubanas se dividen en dos grupos, las de carácter transitorio (los escolares reciben un sistema de provisión de apoyos y pasado algún tiempo transitan nuevamente a la educación general) y las específicas (para alumnos con diagnóstico de Retraso Mental, que se preparan para la vida adulta independiente y egresan, con un nivel correspondiente al $6^{\circ}$ grado ordinario, a un estatus laboral; con ubicación garantizada por la preparación ahí recibida y el Programa Nacional que garantiza el empleo a personas con discapacidad conocido como PROEMDIS.

9. Ambos tipos de escuelas especiales y las escuelas ordinarias trabajan con el currículo básico de la educación general (También las específicas que lo hacen con adaptaciones curriculares más significativas), puesto que existe en el país un único plan de estudio concretado en programas, orientaciones metodológicas, medios de enseñanza y material de estudio iguales para todos, utilizados de forma homogénea simultánea. 
Además de todo ello, estamos de acuerdo con lo planteado en el informe Warnock: una escuela especial constituye la mejor alternativa para educar a ciertos niños. Al menos en el caso de tres grupos: el de los niños con deficiencias graves o complejas -físicas, sensoriales o intelectuales- que requieren instalaciones especiales o un conocimiento docente experto, ya que sería imposible o al menos muy difícil y costoso atenderles en escuelas ordinarias, el de los que presentan trastornos emocionales o de comportamiento graves, con dificultades para establecer cualquier tipo de relación o una conducta tan fuera de lo normal o tan impredecible que causa de problemas en una escuela ordinaria o el de los niños cuyas deficiencias quizás revistan menos gravedad pero que, al ser múltiples, ni siquiera con ayuda les permiten progresar en una escuela ordinaria.

La Educación Especial se ha desarrollado en Cuba a partir de 1959. En el primer decenio pueden referirse los siguientes logros:

- Elaboración de leyes y principios de la enseñanza diferenciada.

- Definición de conceptos, objetivos generales y específicos.

- Determinación de la duración de la enseñanza y niveles.

- Determinación de los planes y programas de estudios, métodos y medios de enseñanza.

- Determinación de los principios de organización y dirección del proceso educativo.

- Extensión de la red escolar.

- Precisión de los métodos de detección, investigación y diagnóstico.

- Organización de la formación y la elevación del nivel técnico del personal docente.

\section{En el segundo decenio:}

- Continuidad en el estudio del análisis del proceso docente-educativo.

- Estructuración del currículo escolar: plan de estudio.

- Selección de los contenidos por especialistas y las disciplinas del currículo.

- Elaboración de principios didácticos y procedimientos metodológicos.

- Organización de cada tipo de escuela (de tránsito, específica o mixta)

- Aumento de matrícula y ampliación de capacidades. 
En el tercer decenio:

- Continúa el perfeccionamiento en la educación especial.

- Asesoría de los especialistas en Europa del este.

- Se inició un programa de construcción de escuelas especiales.

- Profundización del trabajo de los centros de diagnóstico y orientación.(CDO)

De 1989 a Hoy:

- Búsqueda de alternativas para la atención a la población infantil con necesidades educativas especiales.

- Especial énfasis a la labor preventiva y a la preparación laboral.

- Perfeccionamiento del principio martiano de vinculación del estudio con el trabajo.

- Crecimiento del papel de las comisiones de apoyo al diagnóstico de las escuelas especiales.(CAD)

- Perfeccionamiento de la evaluación psicopedagógica.

- Consolidación del trabajo con la familia, la comunidad y las asociaciones de personas discapacitadas.(ANSOC, ANCI y ACLIFIM)

- Demostración sistemática del alto valor social, humano y pedagógico y sus funciones de prevención, tránsito, apoyo e integración.

- Diseño y ejecución de preparación para la vida adulta.

\section{Reconceptualización de la Educación Especial.}

A lo que se ha dado en llamar proceso de reconceptualización de la Educación Especial en Cuba, no es más que a la asimilación del término "necesidades educativas especiales" y de todo lo novedoso que empezó a surgir en el mundo a partir de la Conferencia de Salamanca, respecto a cómo concebir una escuela especial tan centro de recursos y ayuda como parte activa del sistema de escuelas.

Así, podemos exponer las misiones y funciones de la Escuela Especial que se han oficializado en Cuba por la Dirección Nacional de Educación Especial. 
Misiones de la escuela especial:

Desarrollo integral y multifacético de la personalidad del niño acorde con sus posibilidades.

Desarrollar al máximo las capacidades acorde con las particularidades de cada uno.

Preparación para la vida adulta independiente

Integración socio-laboral de nuestros alumnos.

Asimismo se determinaron 4 tipos principales de funciones: Prevención, tránsito, apoyo, integración (Bell, 1998).

Prevención: En el proceso pedagógico que se desarrolla en estas escuelas se coloca un énfasis particular en evitar que las desviaciones y defectos secundarios, terciarios que pueden desarrollarse a partir de los denominados defectos primarios, tengan lugar o que una vez establecidos, se atenúen sus consecuencias.

Tránsito: Proceso que lleva a cabo la escuela para reincorporar los alumnos a sus escuelas de origen. Constituye el proceso y la finalidad más importante de la mayoría de las escuelas especiales cubanas.

Apoyo: Están muy relacionadas con su proyección hacia todo el sistema Nacional de Educación y el trabajo de apoyo que brinda la escuela a los alumnos vinculados con su estrategia de educación familiar y comunitaria. Sustentado esto según el siguiente planteamiento del informe Warnock: algunas de estas escuelas deberían ser desarrolladas específicamente como centro de recursos para uso de todos los profesores del área correspondiente. Ayudarían así al desarrollo curricular y al perfeccionamiento del profesorado. Servirían a padres y profesionales como punto de referencia para el asesoramiento en materia de Educación Especial y actuarían de lugar de encuentro para los propios padres...

Dentro de ellas, la integración más que un tipo de función constituye un fin de la educación especial. Variadas son las acciones que se ejecutan por las escuelas especiales para alcanzar el noble propósito de integrar a la vida social y laboral activa a todos nuestros alumnos. 


\section{Evolución conceptual de la Discapacidad, la CIF y el nuevo concepto}

Hasta hace muy poco se entendió, Discapacidad como:

...restricción o ausencia debida a deficiencia de la capacidad de realizar una actividad dentro del margen que se considera normal para un ser humano...

Esta postura responde al modelo médico, ya que considera la discapacidad como un problema de la persona a consecuencia de una enfermedad, trauma o condición de salud, que requiere de cuidados médicos prestados en forma de tratamiento individual por profesionales; dirigido a conseguir la cura o una mejor adaptación de la persona y un cambio en su conducta.

Para este modelo médico, que se presenta resumido en este esquema:

\section{Deficiencia $\longrightarrow$ Discapacidad $\rightarrow$ Minusvalía}

La deficiencia es la pérdida o anormalidad de una estructura o función psicológica, fisiológica o anatómica del individuo.

La discapacidad, como ya vimos es la restricción o ausencia debida a deficiencia de la capacidad de realizar una actividad dentro del margen que se considera normal para un ser humano.

La minusvalía es la situación de desventaja que surge en un individuo a consecuencia de una deficiencia o discapacidad que limita o impide el desempeño de un rol que es normal en su caso.

Por otro lado, el modelo social de la discapacidad considera el fenómeno fundamentalmente como un problema de origen social y principalmente como un asunto centrado en la completa integración de la persona en la sociedad, ven la discapacidad no como atributo de la persona sino como un complicado conjunto de condiciones, muchas de las cuales son creadas por el contexto/entorno social. 
El manejo del problema requiere actuación social y es responsabilidad colectiva de la sociedad hacer las modificaciones ambientales necesarias para la participación plena de las personas con discapacidad en todas las áreas de la vida social. El problema es más de ideología o de actitud, y requiere la introducción de cambios sociales, lo que en el ámbito de la política constituye una cuestión de derechos humanos. De acuerdo con este modelo, la discapacidad se configura como un tema de índole politica.

A los enfoques médico-biológicos y social le correspondió históricamente la Clasificación reconocida como Clasificación internacional de deficiencia, discapacidad y minusvalía (CIDDM) puesta en marcha en la década de los 70, publicada en el 1980 por la OMS, traducida a 14 idiomas, cuya versión al castellano es la española publicada por IMSERSO en 1983.

Sin embargo, muchas revisiones y propuestas le sucedieron a esa clasificación, la cual fue sustituida durante la aprobación en la 54 $4^{\mathrm{a}}$. Asamblea Mundial de la Salud en el 2001 por la Clasificación Internacional del Funcionamiento de la Discapacidad y de la Salud (CIF)(OMS, 2001). En ella se presenta el nuevo concepto de Discapacidad y además como nueva Clasificación se considera hoy: centro para la Familia Internacional de las Clasificaciones junto a la CIE-10.

La CIF contiene subyacentes los criterios teóricos de un modelo bio-psico- social que entró en contradicción, entre otros, con el concepto de discapacidad vigente hasta ese momento y asociado a la antigua Clasificación conocida como la CIDDM.

El modelo Bio-psico-social

Este modelo ve al ser humano en el centro de las interrelaciones entre las Fundamentaciones Biológicas, las motivaciones psicológicas y los condicionamientos sociales. Plantea que:

El sustrato biológico determina la deficiencia

El sustrato personal la actividad personal

El sustrato social la participación social 
Y todo ello en su interrelación reducen o generan la discapacidad.

Este modelo ve la Discapacidad como:

... limitaciones en la actividad y restricciones en la participación que puede tener una persona como consecuencia de la interacción entre los aspectos inherentes a su condición de salud (Funciones/estructuras corporales), la actividad (limitaciones en la actividad), la participación (restricciones en la participación), relacionados con los factores contextuales (ambientales y personales) que ocasionan resultados negativos.

Como han analizado y demostrado M.A, Verdugo y cols (1995), con este modelo vamos,

De:

La diferencia

La marginación

La estigmatización
A:

Los aspectos comunes

Igualdad

Paridad

Por eso se ha calificado como: más positivo y universal y menos estigmatizante y excluyente.

A la Clasificación Internacional del Funcionamiento, la Discapacidad y los Estados de Salud, la CIF, se le reconoce como contribuciones principales:

- Aplicación del modelo bio-psico-social de la discapacidad

- Introducir el concepto de universalidad para la discapacidad

- Enfatizar en los elementos positivos, no estigmatizantes.

- Establecer un lenguaje común de aplicación universal.

\section{La Discapacidad, una nueva visión y una nueva escuela.}

La Clasificación Internacional del Funcionamiento, la Discapacidad y los Estados de Salud, la CIF, introduce términos que consideramos explicativos del enfoque teórico que ella encierra, ellos son: 
Bienestar: término general que encierra el universo global de los dominios de la vida humana, incluyendo aspectos físicos, mentales y sociales que conforman lo que se puede llamar una "buena vida".

Condición de salud: Término global o baúl para enfermedades, trastornos, traumatismos o lesiones. Puede incluir también otras circunstancias como embarazo, estress y envejecimiento. Las condiciones de salud son codificadas utilizando la CIE-10.

Actividad: Capacidad potencial de una persona en la ejecución de una tarea, en un entorno uniforme.

Limitaciones en la actividad: Dificultades que un individuo puede presentar en la realización de actividades.

Participación: es la implicación en una situación vital. Representa la perspectiva social del funcionamiento. Permite describir lo que las personas hacen en su ambiente/entorno real.

Restricciones en la participación: Problemas que un individuo puede presentar ante situaciones vitales.

Esta concepción refleja un desplazamiento de la ubicación de la discapacidad como consecuencia directa de una determinada deficiencia a una posición universal, ${ }_{2}$ como un todo que incluye el resultado de la interacción de los estados de salud y los factores contextuales; es decir, la CIF está basada en la integración de estos dos modelos opuestos, con un enfoque bio-psico-social; que busca describir, agrupar y clasificar los aspectos del funcionamiento de las personas, que resultan de la interacción del individuo (con una determinada condición de salud) y su entorno(factores contextuales ambientales y personales) desde una perspectiva biológica, individual y social, lo cual puede entenderse mejor en el siguiente esquema: 


\section{Condición de Salud}

(trastorno/enfermedad)

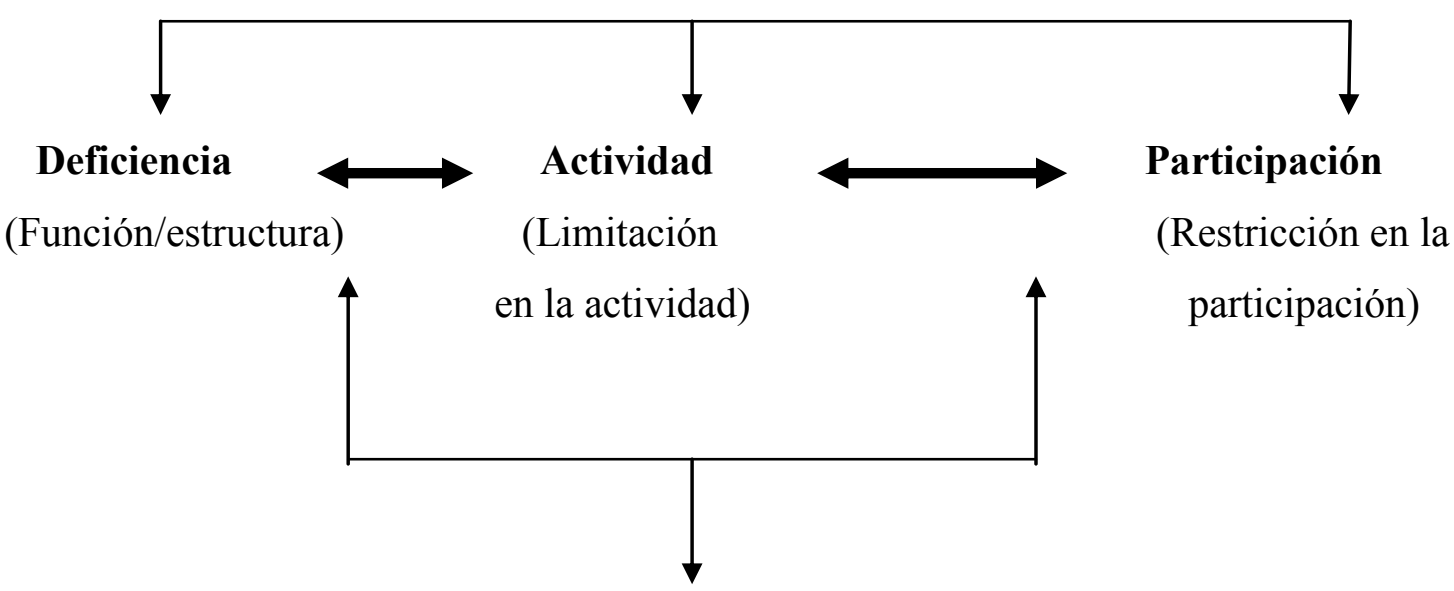

\section{Factores contextuales}
A. ambientales
B. Personales

Se refiere a tres niveles: orgánico, funcional y social.

- Deficiencias: Son problemas en las funciones o estructuras corporales, tales como una desviación significativa o una pérdida.

Las Funciones Corporales son las funciones fisiológicas de los sistemas corporales (incluyendo funciones psicológicas). Las Estructuras Corporales, son las partes anatómicas del cuerpo tales como los órganos, las extremidades, y sus componentes.

Las deficiencias son causadas por problemas prenatales (durante el embarazo), peri natales (durante el parto), o postnatales (enfermedades, accidentes, $u$ otros daños que aparecen después del parto), siendo estas últimas las más frecuentes, lo cual no indica necesariamente que en este momento está presente una enfermedad o que se considere enferma la persona. Las deficiencias derivan en otras deficiencias. Según el curso en el tiempo puede establecerse de forma temporal o permanente. Según el curso de su evolución pueden ser progresivas, estáticas o continuas.

Los niveles orgánico y funcional se concretan una vez determinadas y descritas las deficiencias y sus consecuencias en las funciones y estructuras corporales con lo que se tiene 
resuelto el primer paso para definir "la condición de salud", a lo que sólo resta el análisis de la Actividad y la Participación posibles no sólo a partir de la deficiencia y sus repercusiones corporales sino de los factores, de tipo ambientales y personales, es decir: contextuales. La relación que se da entre estos dos grupos de factores es dinámica y puede nombrarse como relación entre la condición de salud y los factores contextuales. De este modo se trata el nivel social, con su importante papel para entender la discapacidad de manera más universal, resultante de esta interacción dinámica de factores y ya no más como consecuencia directa de una deficiencia.

Todo esto explica que podamos encontrar casos donde el aspecto relacionado con la Deficiencia (ver esquema) sea casi idéntico, y tenga diferencias en la actividad y en la participación, lo que muestra cómo puede ser distinto el nivel de discapacidad, teniendo casi idéntico el nivel de deficiencia, como se ve en el siguiente ejemplo de tres casos:

\begin{tabular}{|l|l|l|}
\hline \multicolumn{1}{|c|}{ Deficiencia } & \multicolumn{1}{|c|}{ Actividad } & Participación social \\
\hline $\begin{array}{l}\text { Mujer Ciega } \\
\text { (25-30 años) }\end{array}$ & $\begin{array}{l}\text { No Cocina, ni limpia, Ni lava, ni plancha, ningu- } \\
\text { na labor hogareña. } \\
\text { No sabe ningún oficio u ocupación } \\
\text { Soltera, vive con sus padres. }\end{array}$ & $\begin{array}{l}\text { No Trabaja } \\
\text { No sale sola }\end{array}$ \\
\hline $\begin{array}{l}\text { Mujer Ciega } \\
\text { (25-30 años) }\end{array}$ & $\begin{array}{l}\text { Realiza las labores hogareñas (Cocina, limpia, } \\
\text { plancha, lava) con ayuda. Tiene habilidades arte- } \\
\text { sanales. } \\
\text { Vive con sus padres y esposo, se niega a tener } \\
\text { hijos por ahora }\end{array}$ & $\begin{array}{l}\text { No Trabaja } \\
\text { No sale sola }\end{array}$ \\
\hline $\begin{array}{l}\text { Mujer Ciega } \\
\text { (25-30 años) }\end{array}$ & $\begin{array}{l}\text { Vive con su esposo e hijo } \\
\end{array}$ & $\begin{array}{l}\text { Trabaja, como re- } \\
\text { cepcionista. } \\
\text { Sale sola usando } \\
\text { bastón. }\end{array}$ \\
\hline
\end{tabular}

Creemos que el ejemplo deja claro que, gracias a la relación que los sujetos tengan con los contextos (independientemente del tipo de deficiencia, sea estructural o funcional y el grado de la misma) estará caracterizada la actividad y la participación siendo éstos más o menos negativos, es decir, teniendo más o menos limitaciones en la actividad y/o restricciones en la participación. 


\section{Una nueva visión, una nueva misión}

Un aspecto importante a considerar es el relacionado con las actitudes de las personas hacia la discapacidad y un análisis de las diferencias que existen, o no, entre las personas implicadas y el resto. Pero, qué son, cómo analizarlas, investigarlas o modificarlas. Entre las tantas definiciones de actitudes vamos a citar, por su concordancia con el tema y el fenómeno concreto de la percepción y el tratamiento a la discapacidad, la de Verdugo, quien plantea que las actitudes podrían ser definidas como un conjunto de predisposiciones que implican respuestas ante una clase específica de objetos o personas y que adoptan diferentes formas; éstas constituyen expresiones de los componentes cognitivos (por ejemplo, información perceptual, estereotipos), afectivos (por ejemplo, sentimientos de gusto o disgusto) y conativos (por ejemplo, intención conductual o conducta en sí misma) de una actitud (Verdugo, 1995).

Entre las múltiples dimensiones de las actitudes vamos a reflexionar sobre los tres componentes que se plantea en la literatura, como estructura categorial de las mismas y que son los componentes: cognitivo, afectivo y conductual.(Triandis, 1971)

1) Componente cognitivo: "Refleja los pensamientos, ideas, creencias, opiniones o percepciones acerca de un objeto actitudinal. Es decir, refleja cómo éste es mentalmente conceptualizado" (Antonak y Livneh, 1988). Es el modo como el objeto actitudinal es definido, este componente suele ser medido utilizando instrumentos tales como las listas de adjetivos y el diferencial semántico.

Respecto a este componente hemos de decir que en Cuba al nivel teórico aunque se proclama en el discurso una actitud de los profesionales a partir de un conocimiento afiliado a la escuela socio histórico cultural encabezada por L.S. Vigotsky, a nivel social y aún dentro de los profesionales, todavía persisten concepciones de la discapacidad como rasgo inherente a la persona y fuertemente ligado a la deficiencia. Las actitudes, por tanto, en su componente cognitivo coinciden con una comprensión de la discapacidad como condición rígida, personal $\mathrm{y}$ en algunos estratos sociales, algunos tipos hasta contagiosas. Investigaciones acerca de la representación social del Retraso Mental, así lo corroboran.

2) Componente afectivo: "Toda la red de pensamientos acerca de las categorías de personas constituye el componente cognitivo de las actitudes hacia los otros. Además, cada 
elemento de esta estructura tiene algún valor afectivo asociado. El afecto se asocia cuando experiencias positivas o negativas ocurren simultáneamente con dicha categoría. De hecho, el ser humano no puede pensar en muchas cosas sin sentir una emoción" (Triandis, Adamopoulos y Brinberg, 1986). La relación se da entre los elementos cognitivos la emoción que se asocie a cada elemento. Puede evaluarse a través de la medición de respuestas del sistema nervioso autónomo simpático (por ejemplo, conductancia de la piel, presión sanguínea, dilatación pupilar, tasa cardíaca).

En Cuba se ha demostrado en investigaciones la presencia de una gran gama de tipos de emociones ligados a las actitudes de las personas, profesionales implicados o no, respecto a la discapacidad. Es claro que de cualquier manera es uno de los aspectos menos investigados y tratados en la formación de profesionales.

3) Componente conductual: Supone la preparación para actuar, la orientación o predisposición, más que el hecho conductual en sí mismo. Un importante conjunto de creencias asociadas a un objeto actitudinal hace referencia a las conductas que pueden ocurrir con respecto a ese objeto. Las opciones en el caso de la conducta social son limitadas: uno puede "ir hacia", "alejarse" o "ir contra" un objeto actitudinal. Si el objeto actitudinal se considera bueno, la respuesta conductual que elicite será el aproximarse, si se considera malo, el evitarlo o el luchar contra él pueden ser opciones adecuadas. El componente conductual suele medirse a través de escalas de distancia social y mediante la observación de los comportamientos que un sujeto manifiesta en una situación en la que está implicado el referente actitudinal. Puede darse rechazo abierto o encubierto. (Verdugo, 1995)

La sociedad cubana, en su lucha por defender criterios de igualdad humana, ha enarbolado programas sociales y científicos en este sentido, así pueden demostrarse resultados de avance cualitativos y cuantitativos hacia la igualdad teniendo en cuenta el sexo, las razas, entre otras. Sin embargo todavía persisten, como en el resto del mundo, en el componente conductual de las actitudes respecto a la discapacidad algunas no tan positivas como las que requiere un mundo mejor que exhiba una real equiparación de oportunidades.

Creemos que, además de lo referido a las actitudes, entre tantos cambios que han de realizarse para el logro real de la equiparación de oportunidades (entendida ésta como el proceso social que tiene la finalidad de que cada uno de los aspectos esenciales de cualquier tipo 
de formación económica y social resulten accesibles para todos; no sólo en lo que respecta a los medios ambientales físicos que se concretan en las actuales barreras arquitectónicas, sino también en lo referente a los servicios sociales y la vida cultural y espiritual) la planificación y provisión de los servicios y apoyos necesarios para personas con discapacidad. No sólo en el ámbito educacional, para las edades temprana y escolar, sino también las que necesitarán para el resto de la vida estas personas. No obstante eso, ya habíamos dicho que nos centraríamos en lo que respecta a la Educación Especial.

Como ya hemos visto, el Sistema Educativo Cubano le reconoce a la Educación Especial, cuatro funciones principales, que ha de cumplir la escuela especial, y un grupo de misiones desde una óptica social, en función del producto que espera la sociedad obtener de este tipo de educación.

Con vistas a realizar un análisis crítico en el que centraremos la propuesta, de manera sintética las repetimos a continuación:

Funciones Escuela Especial Cubana: Prevención, tránsito, apoyo, integración.

Consideramos que dentro de la función de prevención que se le atribuye a la escuela especial hay que empezar a diseñar objetivos de trabajo que garanticen la prevención de limitaciones en la actividad y restricciones en la participación que puedan presentarse por causantes de tipo contextual por mal manejo familiar y/o problemas socio-comunitarios.

La escuela debe ser capaz, como centro de provisión de recursos puestos a disposición no sólo de los alumnos con NEEs sino también de sus familias y entornos, de una manera consciente, planificada y científica, prever y tratar todos los aspectos negativos contextuales que pudieran entorpecer o inhibir el desarrollo de los alumnos, evitando aquellas limitaciones en la actividad y restricciones sociales que son generadas por dichos aspectos. Asimismo debe intentar reducirlos cuando pese a ello hayan aparecido.

Misiones de la escuela especial:

- Desarrollo integral y multifacético de la personalidad del escolar conforme con sus posibilidades.

- Desarrollar al máximo las capacidades acorde con las particularidades de cada uno.

- Preparación para la vida adulta independiente 
- Integración socio-laboral de los alumnos.

- Alcance del nivel mínimo posible de discapacidad, es decir: la menor cantidad posible de limitaciones en la actividad y restricciones en la participación social, acorde con la deficiencia de que se trate.

Con respecto a lo que consideramos que ha de constituirse como una nueva misión de la Educación Especial podría redactarse de varias maneras, lo cual resulta lo menos importante al lado de la envergadura que esta nueva misión alcanza, en el plano social y en el resto, pero sobre todo en las propias personas con discapacidad y sus familias.

Creemos que la preparación para la vida adulta independiente es un fuerte pilar, pero igualmente, que no es suficiente aún tal y como está materializándose este trabajo en la práctica educativa cubana. El tratamiento que se da a los contextos escolar amplio y comunitario no se concreta en la provisión de apoyos o ayudas que satisfagan las necesidades individuales y/o no se concibe planificada y conscientemente en los aspectos más específicos.

Una vía para hacerlo consiste en la implementación de currículos funcionales donde se planteen contenidos y objetivos específicos y acordes con las necesidades educativas especiales de cada escolar, con el cual lograr la realización de aquellas actividades donde tengan limitaciones y disminuir éstas al máximo posible. Asimismo se planificaría contenidos y objetivos sociales (no sólo respecto a la inserción laboral) que garanticen la menor cantidad o ninguna restricción social.

Lo que permita que al momento del egreso, de la escuela especial (en el caso de los que asisten a escuelas específicas y tienen diagnóstico de Retraso Mental) o de la Educación General Politécnica y Laboral (que en algún momento estuvieron en la escuela especial y continuaron necesitando apoyos o ayudas) puedan realizar las actividades que se espera que hagan de manera independiente de acuerdo con su grupo (según edad, sexo y cultura) en todos los entornos.

\section{Familiar:}

- Hogar (relaciones con el resto de los miembros y actividades domésticas y cuidado de sí mismos y del hogar) 


\section{Laboral:}

- Centro laboral (relaciones con el resto del personal, actividades específicas que requieren de la aplicación de habilidades laborales y otras actividades extra laborales como asistir al comedor, reuniones entre otras)

Comunitario: (Actividades de uso y consumo de todos los lugares así como aprovechamiento de los servicios que brinda la comunidad para la satisfacción de cualquiera de las necesidades humanas: espirituales, materiales básicas o no, seguridad, así como las habilidades que se requieren específicamente para cada sitio en cuestión) como por ejemplo los siguientes:

- Comercios (mercados, tiendas, bodegas, carnicerías/pescaderías/lecherías, cafeterías, restaurantes)

- Centros Culturales (teatros, circos, cines, casas de cultura, bibliotecas, museos, galerías de arte)

- Centros religiosos (iglesias, conventos)

- Centros deportivos (estadios, salas de presentaciones de deporte en equipos, terrenos populares de la localidad)

- Centros de recreación (parques de diversiones, acuarios,zoológicos playas, piscinas, campismos, hoteles o villas, clubes, centros nocturnos)

- Centros de Servicios a la Población (lavanderías, peluquerías, Oficinas de correos, , servicios informáticos, telefónicos, oficinas de cobro/pago, terminales de ómnibus/trenes/ aeropuertos)

- Equipos tecnológicos públicos (teléfonos, cajeros automáticos, ascensores)

- Medios de transporte (Transporte local: ómnibus, taxis particulares o estatales, camiones, camionetas, "bicitaxis", carretas con tracción animal; Transporte intermunicipal o interprovincial: taxis particulares o públicos, ómnibus, trenes, aviones, barcos)

- La vía pública. (Calles, avenidas, paseos, carreteras, caminos, parques, otros sitios abiertos urbanos o rurales)

\section{Referencias}


Antonak RF \& Livneh H (1988). Measurement of attitudes toward people with disabilities: Methods, psychometrics and scale. Springfield, IL: Charles C Thomas.

Bell Rodríguez, R. (1996). Sublime profesión de amor. La Habana: Editorial Pueblo y Educación.

Bell Rodríguez, R. (1997). Educación Especial: razones, visión actual y desafios. La Habana: Editorial Pueblo y Educación.

Bell Rodríguez, R. et al. (2001) Educación y Diversidad. La Habana: Editorial Abril.

Gómez Gutiérrez, L. I. Ministro de Educación de Cuba (1998). La atención en Cuba a las necesidades educativas especiales y a niños en edad preescolar. Conferencia. VII Conferencia Científica Latinoamericana de Educación Especial. La Habana.

Gómez Gutiérrez, L. I. (1999). La educación en Cuba. Conferencia Pedagogía 99. La Habana1999.

Gómez Gutiérrez, L. I.(2001). El desarrollo de la educación en Cuba. Conferencia Especial Pedagogía. La Habana.

Organización Mundial de la Salud. (2001). Clasificación Internacional del Funcionamiento, de la Discapacidad y de la Salud «CIF». Madrid: Ministerio de Trabajo y Asuntos Sociales. Secretaría General de Asuntos Sociales. Instituto de Migraciones y Servicios Sociales (IMSERSO).

Triandis, H.C. (1971). Attitudes and attitudes change. Nueva York: Wiley.

Triandis, H.C., Adamopoulos, J., \& Brinberg, D. (1984). Perspectives and issues in the study of attitudes. In Jones, R.L. (1984). Attitudes and Attitude Change in Special Education. Reston, Virginia: The Council for Exceptional Children..

Verdugo, M.A (1994) El papel de la psicología de la rehabilitación en la integración de personas con discapacidad y en el logro de calidad de vida. Siglo Cero 256; 33-42.

Verdugo, M.A. (1995) Personas con discapacidad. Madrid. Siglo XXI. 